\title{
Assessment of the Immunity of Unshielded Multi-Core Integrated Circuits to Near-Field Injection
}

\author{
Ali Alaeldine ${ }^{\# * 1}$, Thomas Ordas ${ }^{+}$, Richard Perdriau *, Philippe Maurine ${ }^{+}$, \\ Mohamed Ramdani \#, Lionel Torres ${ }^{+}$and M'hamed Drissi * \\ \# ESEO-LATTIS \\ 4, rue Merlet-de-la-Boulaye - BP 30926 - 49009 Angers Cedex 01 - France \\ ${ }^{1}$ ali.alaeldinedeseo.fr \\ * IETR - INSA de Rennes \\ 20, avenue des Buttes de Coësmes - 35043 Rennes Cedex - France \\ + LIRMM \\ 161, rue Ada - 34292 Montpellier Cedex 5 - France
}

\begin{abstract}
This paper presents a comparative assessment of the electromagnetic immunity of $\mathbf{4}$ integrated logic cores to near-field injection. These cores, located on the same die, are identical from a functional point of view, but differ by their design strategies. The injection is performed above each core according to the 6 components of the electromagnetic field, using appropriate probes. These results demonstrate that the die and bondwires of an integrated circuit can be sensitive to both magnetic and electric fields, and that some design rules can improve the immunity of integrated circuits to near-field interference.
\end{abstract}

\section{INTRODUCTION}

Within the recent years, many digital and analog integrated circuits (ICs) have become more and more susceptible, due to an increased number of interfaces, higher data rates, decreased node capacitance, and a steady reduction in power supply voltage and, consequently, noise margin. In order to characterize the electromagnetic behavior of these ICs, several measurement methods have been developed by the International Electrotechnical Commission (IEC), in conducted or radiated mode. These methods include near-field [1]; however, this standard is (at the time when this paper is written) limited to emission, although near-field can be used for immunity as well, by injecting power into the pins of an IC through a nearfield probe and observing its behavior according to a given criterion [2] [3]. This method is currently under standardization process by the IEC under the 62132-9 reference.

In this paper, this method is used in order to assess the immunity of unshielded ICs (packaged but without any lid) to electric and magnetic near-field. Furthermore, comparisons among several electromagnetic interference (EMI) protection techniques are achieved using a multi-core IC (CESAME) [4] which had already been used for such evaluations in conducted mode [5]. By minimizing the parasitic influence of packaging, a better comprehension of the immunity of the die itself can be achieved.

The paper is organized as follows. First of all, the internal structure of the CESAME test chip is introduced in Sect. II. Then, Sect. III presents the near-field measurement set-up used in this study. Finally, Sect. IV provides a summary of all measurement results, along with a discussion on the causes of the observed phenomena.

\section{TEST CHIP}

\section{A. General structure}

The integrated circuit used in this study (CESAME) was designed and fabricated by STMicroelectronics in $0.18 \mu \mathrm{m}$ CMOS technology (1.8 V supply voltage), and was initially intended for the validation of low-emission design techniques. It consists of six logic cores which are identical from a functional point of view and are located on the same die, but differ only by their protection strategies. All these cores are based on D flip-flops, a clock tree and standard gates, and are intended to reflect the activity of a typical logic core [4]. Each core includes 240 identical synchronous base cells, and each base cell consists of 400 transistors (5 D-flip-flops, 25 NAND gates and 4 buffers). 4 cores (NORM, RC, NOR and GRID) are built in the global substrate of the IC, while the other ones (ISO and ISV) are built in a local isolated substrate; for the sake of simplicity, only 4 different cores (NORM, GRID, ISO and RC) out of 6 are used.

The IC is mounted in a 144-pin TQFP package. Courtesy of STMicroelectronics, packages with removable lids were used, making it possible to inject a radiated perturbation into a given core with accurate positioning and minimum parasitic influence of the package.

\section{B. NORM core}

The only EMI protection strategy used in the NORM core consists of two small $1.7 \Omega$ series resistors, one on each power supply rail. These resistors, along with the metal and MOS capacitances of the logic core, build up a RC filter, with a high cutoff frequency (about $200 \mathrm{MHz}$ ). 


\section{ISO core}

Another protection strategy is used for the ISO core. This core is embedded in its own local substrate, isolated from the rest of the chip thanks to a triple-well technique (Fig. 1).

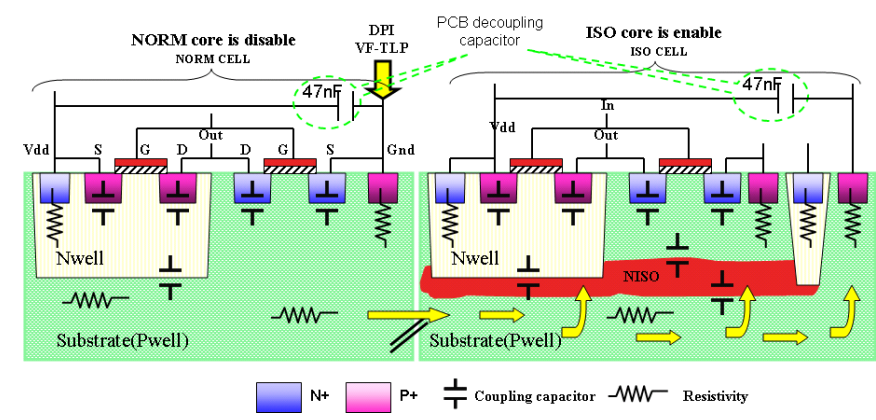

Fig. 1. Comparison between NORM and ISO core architectures

\section{GRID core}

The GRID core, like the NORM core, only uses the series resistors as a protection technique. However, its power supply network is meshed on the lower metal levels (hence the name), thus reducing the total power supply impedance.

\section{E. $R C$ core}

In this core, an additional $1-n F$ integrated decoupling capacitor is included between both supply rails. This distributed on-chip capacitor is made from several poly1/poly 2 capacitors, and increases the area of the RC core by $40 \%$ compared with the NORM core. By lowering the cutoff frequency of the RC filter (about $40 \mathrm{MHz}$ ), this technique makes it possible the power distribution noise arising from multiple drivers switching simultaneously.

\section{NEAR-FIELD INJECTION SET-UP}

The measurement set-up chosen for the characterization of the susceptibility of an IC to near-field interference closely mimics the Direct Power Injection (DPI) set-up. It is depicted in Fig. 2.

For this experiment, the IC is mounted on a $10 \times 10 \mathrm{~cm}$ 4-layer PCB [4], powered by a $9 \mathrm{~V}$ battery through a $1.8 \mathrm{~V}$ regulator, and fed with $20 \mathrm{MHz}$ clock and $10 \mathrm{MHz}$ data signals, in order to ensure normal operation. Then, near-field interference is injected through an adequate probe located at $1 \mathrm{~mm}$ above each core. $1 \mathrm{~mm}$ diameter loops are used for magnetic field injection, while $3 \mathrm{~mm}$ long probes are used for electric field injection. The accurate positioning of the probe is ensured thanks to a picture of the GDS2 layout kindly supplied by STMicroelectronics. Fig. 3 illustrates an electric field injection into the unpackaged IC.

This probe is fed by a RF generator and a power amplifier (rated at $10 \mathrm{~W}$, but delivering up to $46 \mathrm{dBm}$ depending on frequency) through a directional coupler, allowing the measurement of incident and reflected powers through a dualchannel power meter. Therefore, an inductive or a capacitive

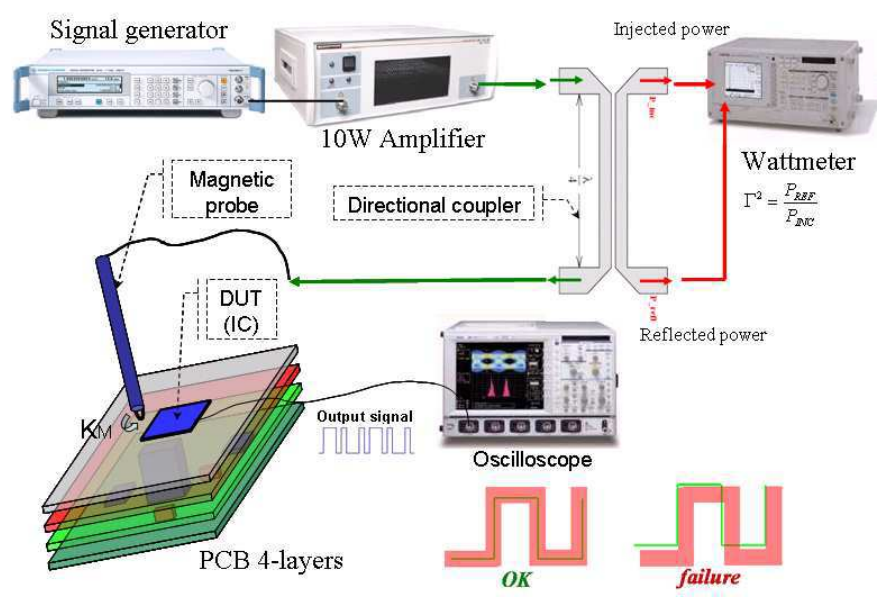

Fig. 2. Near-field injection set-up

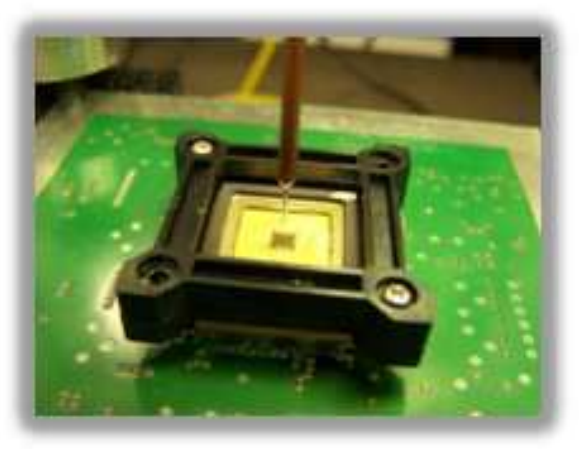

Fig. 3. Photograph of an electric field $\left(E_{z}\right)$ injection

coupling (depending on the probe) is established between with the core. It can be noted that the bandwidth of the amplifier unfortunately limits measurements to $1 \mathrm{GHz}$.

The immunity criterion is an ubiquitous one issued from CAN transceiver testing [6] and already used in many papers. The operation of the IC is considered defective as soon as the ripple of the output amplitude crosses the $20 \%$ margin or the jitter of the output signal is higher than $10 \%$.

\section{RESULTS AND DISCUSSION}

Measurements were performed on the 4 cores (NORM, ISO, GRID and RC) for each component of the electromagnetic field $\left(E_{x}, E_{y}, E_{z}, H_{x}, H_{y}, H_{z}\right.$ ), from $10 \mathrm{MHz}$ to $1 \mathrm{GHz}$ in $10 \mathrm{MHz}$ steps. For each frequency, the injected power triggering a malfunction, according to the aforementioned criterion, was recorded and used for subsequent plots. During this experiment, it was observed that, below approximately 200 $\mathrm{MHz}$, interference has an influence on ripple whereas, above this limit, jitter is affected.

The first result obtained demonstrated that all cores are immune to a $46 \mathrm{dBm}$ injection (upper limit of the amplifier) in normal magnetic field $\left(H_{z}\right)$ at any frequency up to $1 \mathrm{GHz}$. Therefore, no figure is supplied for this component. Fig. 4 to 8 plot the results obtained for the other 5 components of the 
field.

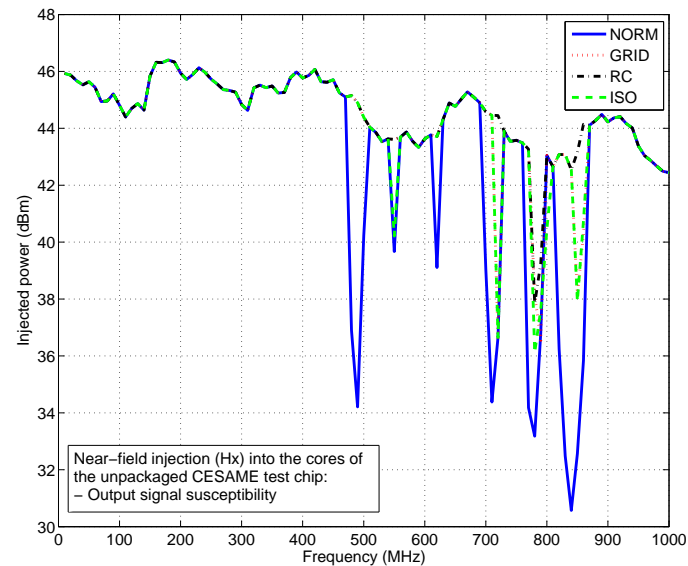

Fig. 4. Immunity of the 4 cores to the $H_{x}$ field

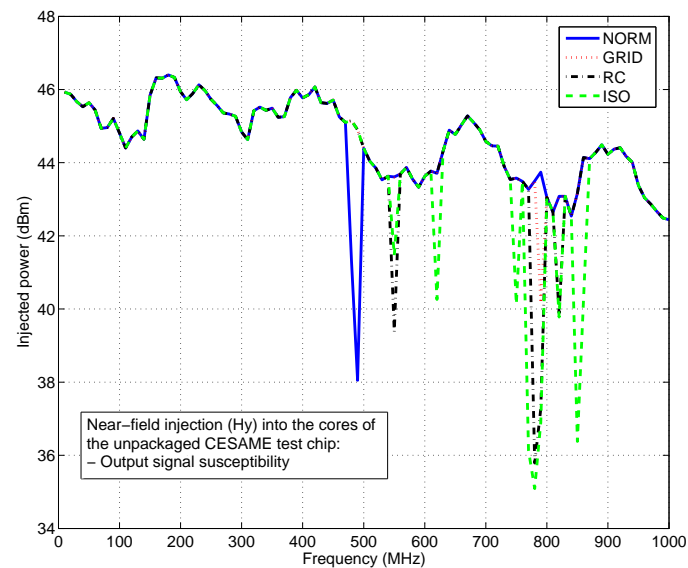

Fig. 5. Immunity of the 4 cores to the $H_{y}$ field

The high immunity of all cores to the $H_{z}$ field comes from the small dimensions of horizontal metal loops on the die; in fact, the voltage disturbance induced in these loops by the probe is very low.

The NORM core, which is the most susceptible core among those studied, is less immune to $H_{x}$ than to $H_{y}$. The explanation may come from the coupling between the magnetic field emitted by the probe and the power supply bondwires: when the loop is parallel to these bondwires, field lines induce a much higher voltage in the power supply than in the case of a perpendicular loop; these disturbances are then reinjected into the die in conducted form. The fact that the $\mathrm{RC}$ core is the most immune to magnetic fields may confirm this assumption and explain why this result matches the one observed in direct power injection (DPI) [5]. Furthermore, it is likely that the die itself is not susceptible to tangential magnetic fields, since the

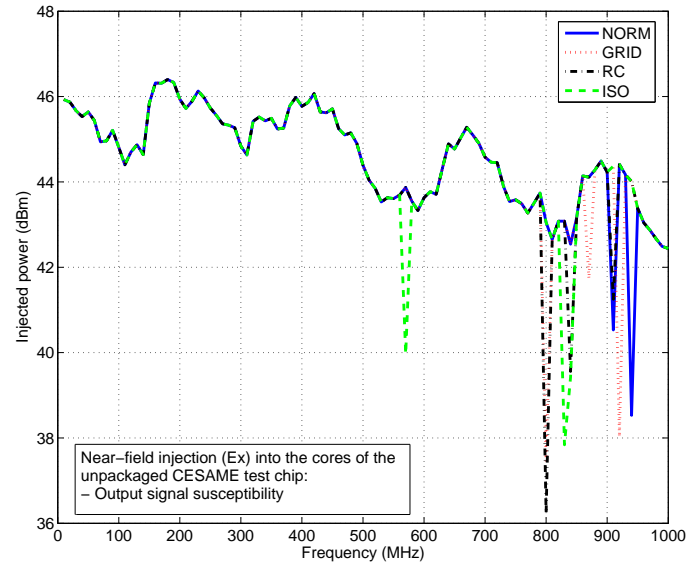

Fig. 6. Immunity of the 4 cores to the $E_{x}$ field

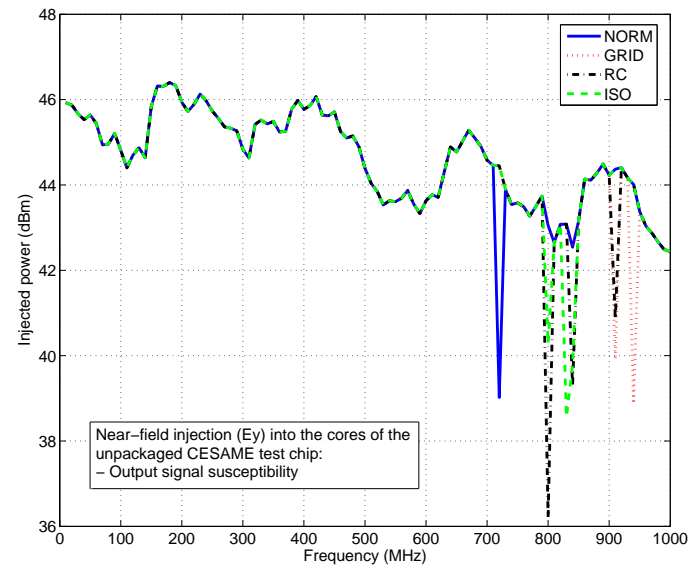

Fig. 7. Immunity of the 4 cores to the $E_{y}$ field

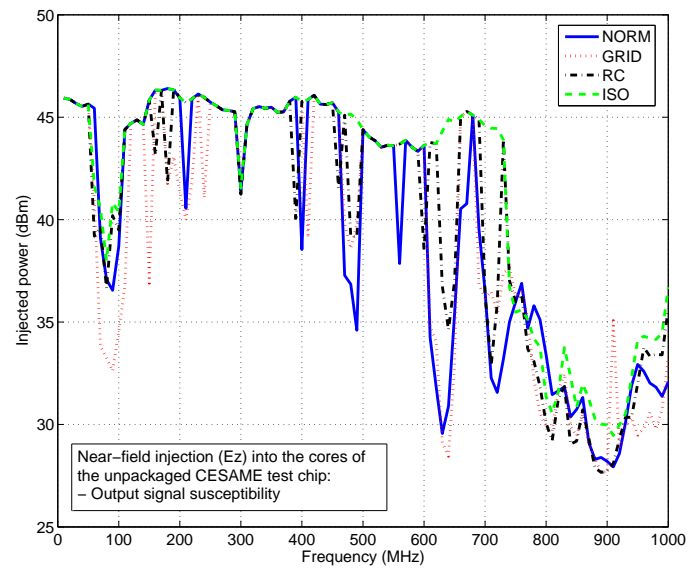

Fig. 8. Immunity of the 4 cores to the $E_{z}$ field 
area of vertical loops (in the power distribution network) is very small.

In general, all cores are more susceptible to electric fields than to magnetic fields. In fact, integrated circuits use "body ties", namely substrate to GND or N-well to VDD contacts, designed to minimize voltage gradients in the substrate. These body ties build up large metal zones with a very low impedance towards the power supply, onto which an electric field can couple itself quite easily (and to a greater extent in the case of a vertical electric field). This capacitive coupling may also explain the difference observed with the GRID core; the capacitive coupling with the meshed power supply network causes new resonance and antiresonance frequencies.

Both RC and ISO cores bear almost the same immunities to $E_{x}$ and $E_{y}$; however, as far as the $E_{z}$ field is concerned, the ISO core is the least susceptible. From this result, an assumption may be made. The vertical electric field is mainly coupled to the body ties, and its reinjection into the ISO core is minimal due to the lower equivalent capacitance of its substrate; conversely, the tangential electric field also targets bondwires and injects the disturbance into the core through the power supply network, hence being filtered by the integrated decoupling capacitance.

In order to identify the contribution of the probes themselves to these results, the standing wave ratios (SWR) of these probes were measured with and without the board under test, with no significant differences. Fig. 9 and 10 depict the SWR of the electric probe and the magnetic probe.

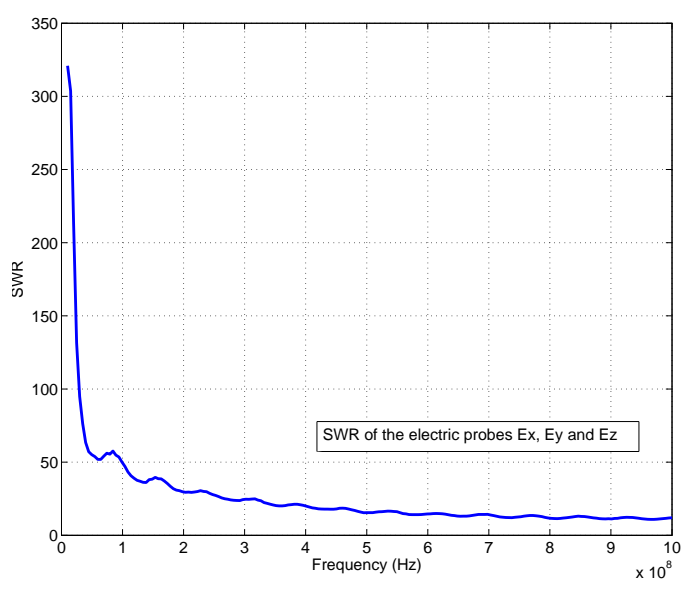

Fig. 9. SWR of the electric probe

As can be seen, the SWR decreases from 30 to 18 (E probe) or 20 to 12 (H probe) in the $0.4-1 \mathrm{GHz}$ range, but rather steadily and with only small variations with respect to its absolute value. Consequently, the steep immunity "valleys" observed in the previous figures are not due to the probes themselves.

\section{CONCLUSION}

In this paper, the effect of near-field electromagnetic injection on unshielded ICs was studied. In particular, comparisons

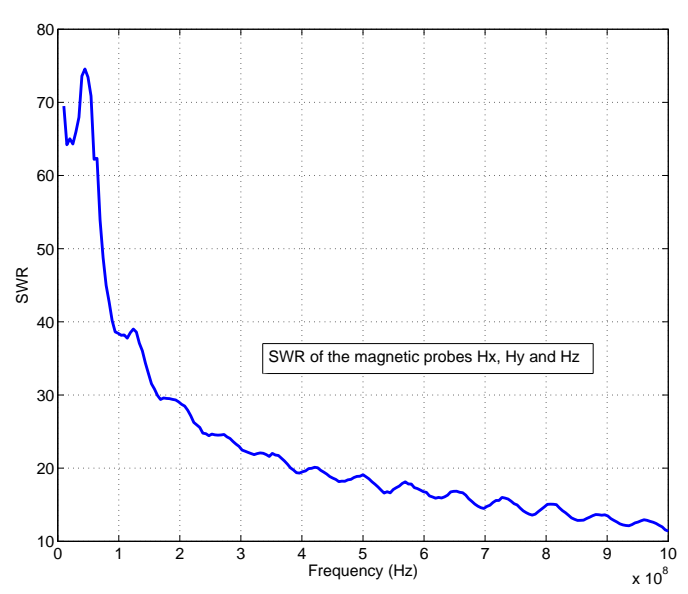

Fig. 10. SWR of the magnetic probe

among different EMI protection strategies on logic cores were assessed. It was demonstrated that integrated circuits are sensitive not only to magnetic field, but also (and to a greater extent) to electric field; moreover, the susceptibility of an integrated circuit to radiated emission does not come only from its bondwires and its package, but also from the die itself. In most cases (except for normal electric field), the integrated decoupling capacitor was proven to be a very valuable technique for susceptibility reduction, as it had been demonstrated in the past for direct power injection (DPI). In order to evaluate the influence of packaging with better accuracy, comparisons will be performed with commercially packaged integrated circuits (the package acting like an electromagnetic shield). It should then be possible to enhance the existing electrical models of near-field injection with better coupling models.

\section{REFERENCES}

[1] IEC EMC Task Force. IEC61967-3 : Integrated circuits, measurement of electromagnetic emissions, $150 \mathrm{kHz}$ to $1 \mathrm{GHz}$ - part 3: Measurement of radiated emissions - surface scan method. Technical report, IEC, June 2005.

[2] J.J. Laurin, S.G. Zaky, and K.G. Balmain. EMI-induced failures in crystal oscillators. IEEE Transactions on Electromagnetic Compatibility, 33(4):334-342, November 1991.

[3] A. Boyer, S. Ben Dhia, and E. Sicard. Characterisation of electromagnetic susceptibility of integrated circuits using near-field scan. IET Electronics Letters, 43(1):15-16, January 2007.

[4] B. Vrignon, S. Ben Dhia, E. Lamoureux, and E. Sicard. Characterization and modeling of parasitic emission in deep submicron CMOS. IEEE Transactions on Electromagnetic Compatibility, 47(2):382-387, May 2005.

[5] A. Alaeldine, N. Lacrampe, J.L. Levant, R. Perdriau, M. Ramdani, F. Caignet, M. Bafleur, E. Sicard, and M. Drissi. Efficiency of embedded on-chip EMI protections to continuous harmonic and fast transient pulses with respect to substrate injection. In IEEE International Symposium on Electromagnetic Compatibility (EMC2007), Hawaii, USA, July 2007.

[6] IEC EMC Task Force. IEC62228 : Integrated circuits - EMC evaluation of CAN transceivers. Technical report, IEC, February 2007. 